\title{
ASSORTMENT OF ONTOLOGIES USEFUL IN NATURAL LANGUAGE PROCESSING
}

\author{
Goonjan Jain ${ }^{1}$, Achal Bansal ${ }^{2}$ \\ ${ }^{I}$ PhD Research Scholar, SCSS, JNU, New Delhi, India \\ ${ }^{2}$ Tech Lead, Fiserv, Open Solutions Division, Gurgaon, India
}

\begin{abstract}
Since the field of NLP has a long history, the literature in this field is growing fast through the recent years. Different knowledge bases or ontologies are available that help us capture different aspects of natural language. There is a huge variety of ontologies that are available either freely or are licensed. Each of these has some new information to offer. But to the very core, each ontology offer a certain kind of knowledge supporting its underlying principle like common sense knowledge, knowledge about purpose of an artifact etc. In this paper, we have tried to collate different ontologies that offer us such diversified knowledge with varied underlying principle which have proved to be helpful in understanding the natural language.
\end{abstract}

Keywords: NLP, Ontologies, WordNet, ConceptNet, Cyc, BableNet, VerbNet, FrameNet, PropBank.

\section{INTRODUCTION}

Ontology, literally means science of classification and is a formal naming and definition of the types of the entities, their properties, and the interrelationships that exists for a particular domain of discourse. Different fields like Artificial Intelligence, Biomedical Informatics, and industrial informatics create and maintain their own ontologies to limit complexity and to organize information.

Common components of ontologies include:

- Primitives: Basic level of entity representation.

- Classes: Collection of objects of similar types.

- Attributes: Objects or classes can have properties, features, characteristics, or parameters that comes under the purview of attributes.

- Relations: specifies the ways in which classes and individual entities can be related to one another.

- Constraints: Restrictions imposed on the input to be formally accepted.

- Inference Engine: This include the rules to generate new information using the exiting one using logical inference.

- Events: Happening of event is associated with changing of attributes or relations.

\section{Types of Ontologies:}

1. Domain Ontology: A domain ontology contains knowledge from a particular domain. Terms which have more than one meaning then a domain ontology will represent the predominant meaning if the term belonging to the ontology. For example, the word mouse has many different meanings. Computer ontology will model it as device, while animal world ontology would model as an animal.

2. Upper Ontology: An upper ontology describes general concepts that are same across all knowledge domains. It describes general entities not belonging to any specific domain as a hierarchy of entities and associated rules.

3. Hybrid Ontology: Hybrid ontology is a combination of upper and domain ontology. E.g. Gellish ontology.

We have discussed only upper ontologies in this paper.

\section{Ontology Learning}

Ontology creation is a not only collecting data about some domain but is a systematic process to gather data and categorize it properly for easier and faster access. Ontologies are created through automation or semiautomatically by extracting terms pertaining to a particular domain and the relationships existing between these terms extracted from a corpus of natural language text. Manually building ontologies is extremely tedious and time consuming task, so efforts are being made to automate the process.

\section{UPPER ONTOLOGIES USEFUL IN NLP}

1. WordNetMiller et al. [2][5][6] proposed WordNet, an online lexical database, in whichnouns, verbs, adjectives and adverbs have been hierarchically arranged in terms of synonyms called synsets. Each synset consists of a list of synonymous words or collocations, and represents a sense. For an example, one sense of word plant is a living organism and the corresponding synset include words like flora and plant life. And the other is building for carrying on industrial labour and the synset includes words like works and industrial plant.

Synsets are connected by different relationswhich include -

- Hypernymy/hyponymy (superordinate/subordinate). For example, for the noun child the inherited hypernym output is -juvenile, person, organism, living thing, whole unit, object and physical entity. The inherited hyponym 
output include concepts like child-prodigy, foster-child, orphan, poster child etc.

- Antonymy refers relationship where words have opposite meaning. For example, deposit $=>$ withdrawal.

- Entailment refers relationship between two verbs where a verb A entails verb B if truth of B follows logically from truth of A. For example, eat entails chew.

- Meronymy/Holonymy is a part-whole relationship. For example, body -- HAS PART:head, neck, torso, arm, ..., leg).

Nouns and verbs are organized into hierarchiesbased on the hypernymy/hyponymy relation between synsets. For example, boy $=>$ man, adult male $=>$ male, eat=>consume etc. Similarly, words are related with synsets based on their syntactic category using different relations.

WordNet has been populated manually. There are more than 117,000 synsets in which more than 155,000 unique words that have been categorizedand can be accessed either using GUI-based access or can be downloaded and used thereafter. The information contained in it is highly useful and has been mapped into several other ontologies. Its applications are in the area of Information Retrieval and Extraction, Word Sense Disambiguation, Document structure and categorization, Audio and Video retrieval, and Semantic Distance calculation.[1] Looking at the advantages and the enormous amount of information that is contained in WordNet, now WordNet is being developed in many languages. Like HindiWordNet, IndoWordNet, EuroWordNet etc. It is available in sixty languages worldwide.

EuroWordNet is a collection of WordNet based semantic networks for different European languages that are interconnected with interlingual links. The original EuroWordNet project dealt with Dutch, Italian, Spanish, German, French, Czech, and Estonian. HindiWordNet is another ontology based on WordNet that collates different lexical and semantic relations between the Hindi words. The lexical information is stored in the form of word meanings.

2. ConceptNet [7] is a large semantic network of common sense knowledge and practical reasoning toolkit. It is a project targeted at acquiring and saving assertions about everyday life. It constitute knowledge in the form of assertions about everyday life which comes under the purview of common sense. The Knowledge Basewas developed through Open Mind Common Sense (OMCS) project in which data was collected from volunteers online using free text or templates.

The basic primitives of representation in ConceptNet 5.0 are concepts. Concepts may be things or actions, usually in phrasal form. The concepts in a ConceptNet can be single words, phrases or senses of words. Concepts can be noun, verb, adjective or adverbial phrases. Assertions are statements about concepts expressed by a relation between two concepts. For example, "a car is used for driving" expresses an assertion, where the relation $/ \mathrm{r} /$ UsedFor connects /c/en/car to /c/en/drive.Justification is a number that gives the final tally of votes in favor of or against the assertion which have made it either true or false. A positive justification indicates that a statement is probably true, whereas a negative justification indicates that it is probably false. A zero indicates a 'don't-know' case. The representation is in flat file format with indices built on top of JSON representation. It has 21 inter-lingual relations and many more relations that are language-specific. The Semantic roles in ConceptNet are categorized as those related to object, events and function. It uses tagged URIs to uniquely identify every concept and assertion in the knowledgebase. For example, /c/ represents a 'concept', /r/ is a multi-lingual relation, /en/ represents an assertion in English language, and $/ \mathrm{n} /$ indicates a noun phrase, and so on. It has found its utility in topics like text summarization, casual projection, document classification etc.

The knowledge in ConceptNet depends heavily on internet users for justifications i.e., determining if an assertion is true or false. If the sum total of the justifications is positive, the assertion is considered as true and false otherwise. The truth and falsity of statements is totally dependent on the intentions of the internet user. This means that a set of internet users can make an actually true statement false, and vice-versa. URIs are overloaded, i.e., there are multiple assertions with the same URI, making the knowledgebase more voluminous.

3. PurposeNet[14] - This ontology enlists the purpose, an artifact is used for and thus named PurposeNet. This is a comprehensive Knowledge Base that address all possible information requirements about artifacts with 'purpose' as the underlying principle. Objects are described in terms of four major types of attributes: dharm (intrinsic property), svabhav (common attributes), rup (perceivable attributes), gun(indirectly perceivable). Purpose is a very significant information about artifacts. An artifact is made in order to serve a particular purpose. Various characteristics of an artifact and activities that can be performed with the artifact depends upon the purpose for which it is created. The purpose of an object is given by the action it helps to perform. Every object also has a structure which helps in fulfilling the purpose of object. In this ontology, artifacts have been described as being constituted of relationships and features.

4. HowNet [16] is a $\mathrm{KB}$ developed with an underling principle that all matter - physical and metaphysical, is in constant motion, and is ever-changing in a given time and space. With time, things evolve from one state to another and this change leads to change in their attributes as well. For example, when a person grows, 'eye sight' (physical) becomes 'weak', and 'character' (metaphysical) changes to 'mature'. The units for manipulation and description in HowNet are - a) thing b) part c) attribute d) time e) space f) attribute-value g) event.

i) All matter is part of something else, and vice-versa. This is basically determined by the point of reference.

ii) Space is represented by Up, Down, Left and Right. 
iii) Time is represented as Past, Present and Future. iv)Attribute determines the similarity and differences between objects. There is no object that does not have attributes. In case of humans, natural (e.g., height) and social (e.g., nationality) attributes exist. Under specific conditions, attributes are more important than the host itself. For example, in the absence of a hammer, any hard and heavy object tool would do.

HowNet is a graph with Inter-Concept and Inter-Attribute relations. The relations defined are - hypernymy-hyponymy, synonymy, antonymy, converse, part-whole, attribute-host, material-product, agent-event, patient-event, instrumentevent, location-event, time-event, value-attribute, entityvalue, event-role, concepts co-relation. About 1500 features and event roles have been represented, preserving word order.

5. LifeNet(2003) [12]-LifeNet is another knowledge base to capture common sense. However, it restricts its propositions to first-person model of human experience represented as undirected graph.This knowledge base represents knowledge that relates80,000 first person propositions with 415,000 links between these propositions.It has an associated inference engine that is based on probabilistic belief propagation. The goal for developing an ontology like LifeNet is to develop a base for commonsense reasoning about a person's context. LifeNet focuseson aspects of person's situation like what they said, when they came, where are they are and so on.

The assertions included in LifeNet are part of Open Mind Common Sense corpus which has already been fully consumed in ConceptNet and so we can say that it is kind of redundant in the knowledge that it contains. But it is quite handy while dealing with text in first-person perspective and also the reference engine helps us to deduce much more than ConceptNet can do.

6. MindNet[9]-MindNet is a lexical knowledge base which is more than a static resource. It represents a general methodology where semantic information is extracted from natural language text. It acquires knowledge, define structure, and access the semantic information from natural language text.It is constructed automatically from the definitions and example sentences in two machine-readable dictionaries (MRDs).

MindNets for an input text are produced automatically. The process takes the input text and breaks into sentences.Each sentence is parsed individually to build a semantic dependency graph. Then these graphs are aggregated into a single large graph, where probabilistic weights are assigned to subgraphs based on their frequency in the corpus. The project also has a mechanism for searching and sorting the graphs as well as measuring the similarity of paths in a MindNet. The automatic acquisition process of knowledge in MindNet makes it a one good prospect for acquiring world knowledge on the scale needed to support commonsense reasoning.
A web-based interface has been provided which facilitates browsing of MindNet structures as well as relations, called MNEX (MindNet Explorer). MNEX displays paths in the form of links that connects words that the user enters. The end points of links connect words within either a single semrel structure or by combining fragments from multiple semrel structures. The paths thus generated between the words are weighted for comparison. This resource is created automatically thus, quickening the process of building domain lexical resources. Althoughthe resource is built quickly but with lesser accuracy.

7. SUMO [1] - The Suggested Upper Merged Ontology (SUMO) and its domainontologies are concept-based. It consists of SUMO itself, the Mid-Level Ontology (MILO), and ontology of eighteen domains like communications, countries and regions, engineering components, government weapons of mass destruction and so on. Each of these domain-specific ontologies has a separate set of semantic relations. SUMO is written in the SUO-KIF language, whereas, the individual ontologies have been implemented in CLIPS. It has been developed by manual effort. It has 20,000 terms and approximately 70,000 axioms (all domain ontologies combined). It is richly axiomatized. All terms are formally defined. SUMO has been mapped to the entire WordNet lexicon. The ontology is available for free download. It provides language generation templates for Hindi, Chinese, Italian, German, Czech, and English. It also provides GUI support for browsing and editing. It also has an independent inference and ontology management system.

The SUMO ontology suffers from a lack of a single, consistent underlying principle for design of the ontology. The categorization of the base hierarchy into numeric, temporal, mereotopology and class theory looks to be a random selection. For example, entities of part_of relationship have been clubbed with the subtype. Also, artifacts are defined under corpuscular objects, (whereas there are many artifacts, for example Screw, that are atomic structures). Process and Object are placed hierarchically below physical. Many such irregularities are found in the Upper ontology design. Also, implementation-wise, no rigid semantics are defined.

Singular and Plural elements - for example - cognitive agent (a singular) and group (its plural) are placed in two different hierarchies. Inheritance of concepts, which is primarily done by the is_a hierarchy, seems to have been ignored.

8. FrameNet [3] is a concept-based resource for actions and states, represented by verbs. The Knowledgebase is based on annotating examples of how words are used in actual text. It is a dictionary of 10,000 word senses. The underlying principle used is 'Frame Semantics'. A Semantic Frame is a description of a type of event, relation, entity or participant. The basic concepts in FrameNet are - the frame, which gives the name of the frame with a textual description of the concept it represents, and frame elements (FEs) - which represent semantic roles - like time, place, relatives etc. For example, the Apply_Heat frame has the FEs - Cook, Food, 
Container and Heating_Instrument. Example sentences are used to prove that the Fes fit exactly around the word that evokes the frame. For example, [Cook The boys]...grill [Food their catches] [Heating_Instrument on an open fire.].It has been developed manually. Polysemous words are represented by several lexical units, 800 semantic frames, and over 1,70,000 manually annotated sentences. FrameNet annotations are triplets consisting of - Frame Element Name(eg. Food), a Grammatical Function (eg. Object), and a Phrase Type (eg. Noun Phrase). The annotation has been implemented using XML. It has a GUI for user-friendly access. Frame elements are related through annotation. It also gives valences - the number and position of frame elements within example sentences. It has been mapped by several ontologies like VerbNet. This resource is available for free download. FrameNet is used as a teaching material for semantic classes, multilingual lexical basis, Interlingua for machine translation, and training data for Questionanswering, and Information Retrieval.

FrameNet does not have an inbuilt inference engine or a module for consistency checking. The ontology is not domain-specific. The information provided by FrameNet on events lacks information on precondition, results and subaction, which are integral to any action-related reasoning. It shows syntactic roles, but, very few example sentences are available. Core and NonCore frame elements are considered as semantic roles. But, different semantic roles are considered as Core and Noncore based on the frame, which is confusing. For example, for Being_Born frame, the core frame element is child, whereas, for Commerce_goods-transfer frame, the core frame elements are buyer and seller.

9. VerbNet[8] is a verb lexicon which is linked with other ontologies like WordNet and FrameNet. The data is domainindependent and is organized hierarchically.Each verb or predicate has an individual verb class which describes each verb. Each verb class contains thematic roles associated with the verb and selectional restrictions to be imposed on the arguments. Each verb class also contains information about frames that consists of syntactic description and semantic predicates. Syntactic description contained in VerbNet class for a verb depicts the possible surface realizations of the argument structure. Semantic or selectional restrictions puts a check on the types of thematic roles allowed by the argumentssuch as animate, human, organization for a given verb. For an example, agent of verb 'eat' should be animate. It can also restrict the syntactic nature of the constituents to be associated with the thematic role. An event variable $\mathrm{E}$ is associated with each semantic predicate that shows the truth value of the event for the predicate. Start (E) gives the truth value for the predicate for preparatory stage, during $(\mathrm{E})$ shows truth value for the culmination stage, and end (E) for the consequent stage. VerbNet has 2205 assertions. It has a GUI for KB access. It has been implemented in XML format to enable access over the web. This resource is available for free download.

VerbNet does not have an inbuilt inference system. Consistency check module is absent in VerbNet. The ontology is non-domain specific. It is used as a basis for parameterized action representation, and robust semantic parsing. VerbNet does not distinguish between the three kinds of results of actions - results (that are expected outcomes), side effects (those that are unintentional), and wear and tear (that incurs to the artifact as a result of an action performed by and on it). Further it does not cover all possible domains and commonsense usage. For example, the artifact is defined as a concrete physical object. Also, artifact has only three categories - machine, garment, and tool; all other categories are missing.

10. PropBank [10][11]- The Proposition Bank or PropBankis a semantic role annotated knowledge base. Penn Treebankis used to showcase the predicate-argument relationship for all the predicates is the corpus.Its main focus is to make available argument structure of verbs.It covers every instance of every verb in the corpus and allows representative statistics to be calculated.One of the goals to develop such an extensive knowledgebase was to provide consistent argument labels irrespective of the syntactic structure of the same verb.

[Rohan]ARG0 broke [the glass]ARG1

[The glass]ARG1 broke.

The arguments of the verbs are labeled as numbered arguments: ARG0, ARG1, ARG2, and so on. Each verb or predicate has an individual frame file which contains different argument structure for the verb in the corpus. The frame file gives both semantic and syntactic information about each sense of the predicate encountered in PropBank annotation. The relationship between numbered arguments and semantic roles is unique for all the predicates, so every frame file shows how the two are related for the given predicate.Some of the arguments are required for the valency of a predicate and others are those arguments that are used very frequently along with the predicate.Generally, numbered arguments correspond to the following semantic roles, though they may vary based on the usage of the predicate:

Table-1: List of common arguments in PropBank

\begin{tabular}{|l|l|}
\hline ARG0 & Agent \\
\hline ARG1 & Patient \\
\hline ARG2 & Instrument, benefactive, attribute \\
\hline ARG3 & Starting point, benefactive, attribute \\
\hline ARG4 & Ending point \\
\hline ARG5 & Modifier \\
\hline
\end{tabular}

In addition to numbered arguments, another task of PropBank annotation involves assigning functional tags to all modifiers of the verb, such as manner (MNR), locative (LOC), temporal (TMP) and others.

This hand annotated corpus in the form of PropBank is useful in development of better domain-independent language understanding systems. This also makes its utility count by helping in understanding the syntactic alternations 
that take place in the language. This level of semantic representation is important for applications including information extraction, question answering, and machine translation.

The resource is useful to the extent that tagging the sentence with semantic roles, we refer to PropBank. Also there are many tools in the market that tag sentences the thematic roles of the words in the sentence for a given verb by using PropBank annotations. Looking at the pool of opportunities it opens if we know the semantic role, it has been developed in various languages round the globe so like Hindi-Urdu, Chinese, Arabic, Finnish to name a few.

11. Cyc[14] is a lexicalontology that attempts to assemble the $\mathrm{KB}$ of everyday common sense knowledge, with the goal of enabling Artificial Intelligence applications to perform human-like reasoning. Its components are Constants, Collections, Truth functions and Functions. In Cyc, the word has noun, verb, adjective, and adverb forms. Cyc-NL's clausal semantics is "verb-driven". Verbs are stored in the lexicon as templates for their translation into a formal programming language called $\mathrm{CycL}$ which is based on predicate calculus. It has been developed manually. The knowledge base $(\mathrm{KB})$ is divided into microtheories (Mt) which is a collection of concepts, and facts belonging to a particular domain. There are over one million humandefined assertions, rules, or common sense ideas in the knowledge base. The system has been furnished with the ability to communicate with end users in natural language. Its inbuilt inference engine assist with the knowledge formation process via machine learning. For example, a small piece of knowledge are "Dog is aanimal" and "Animals die eventually". When we ask the system whether dogs die, the inference engine of the system comes into action and conclude the correct answer. Cyc is used in
Medical Research analysis, Counter Terrorism analysis, financial analysis, and Network Intrusion protection.

CycL is very complex and difficult to learn. There is no API available for application development using CycL. The complete version of Cyc is unavailable. The assertions in OpenCyc are non-domain-specific. There are scalability issues resulting in its inference system being slow and unreliable. The KB has been entirely built manually and has not been mapped to any other ontology. It is very difficult to merge the KB with any other existing ontology.

12. BabelNet[13] is a multilingual lexicalized semantic network and ontology developed at the Linguistic Computing Laboratory in the Department of Computer Science of the Sapienza University of Rome. It was generatedautomatically by linkingWikipedia to WordNet. The integration of these two knowledge sources was performed by automatic mapping. The ontology is multilingual with more than 270 languages under its umbrella, it fixes lexical gaps using statistical machine translation for resource-poor languages. The result of this merger is an "encyclopedic dictionary" in which information is collated in the form of concepts and named entities connected to each other with a number of semantic relations. To spread the domain of knowledge, it has also linked data from sources like OmegaWiki, Wiktionary etc. The words in BabelNet are arranged as Babel synsets which are sets of synonyms in different languages. Each synset is associated with a gloss which gives a short definition in many languages.

It also contains translations obtained from sense-annotated sentences.BabelNet is fully integrated with a state-of-the-art multilingual disambiguation and entity linking system, called Babelfy.

Table-2: Summary of ontologies discussed in the paper

\begin{tabular}{|c|c|c|c|c|c|c|c|c|c|}
\hline Ontology & $\begin{array}{l}\text { Primitive of } \\
\text { Design }\end{array}$ & $\begin{array}{l}\text { Domain } \\
\text { Specific } \\
\text { Design? }\end{array}$ & $\begin{array}{l}\text { Consistency } \\
\text { Check for } \\
\text { Input Data }\end{array}$ & $\begin{array}{l}\text { Inbuilt } \\
\text { Inferenc } \\
\text { e System }\end{array}$ & $\begin{array}{c}\text { Availability } \\
\text { for } \\
\text { Download }\end{array}$ & $\begin{array}{l}\text { Mapping to } \\
\text { other ontology }\end{array}$ & $\begin{array}{l}\text { Language } \\
\text { used for } \\
\text { resource } \\
\text { creation }\end{array}$ & $\begin{array}{l}\text { GUI for } \\
\text { access }\end{array}$ & $\begin{array}{c}\text { Lexical/Se } \\
\text { mantic/Bo } \\
\text { th }\end{array}$ \\
\hline WordNet & Synset & No & Yes & No & Yes & $\begin{array}{c}\text { Mapped by } \\
\text { other Ontolgoy }\end{array}$ & XML, OWL & Yes & Both \\
\hline Сус & Microtheories & No & Yes & Yes & $\begin{array}{c}\text { yes - } \\
\text { OpenCyc }\end{array}$ & Nil & Cycl. & Yes & Both \\
\hline SUMO & Concept & Yes & Not Complete & Yes & 1 Domian & WordNet & SUO-KIF & No & Both \\
\hline FrameNet & Frame & No & Yes & No & Yes & $\begin{array}{c}\text { Mapped by } \\
\text { other Ontolgoy }\end{array}$ & OWL, XML & Yes & Both \\
\hline VerbNet & Verb & No & No & No & Yes & $\begin{array}{l}\text { WordNet, Xtag, } \\
\text { FrameNet }\end{array}$ & XML & Yes & Both \\
\hline HowNet & Changing states & No & Yes & Yes & Yes & $\begin{array}{c}\text { Mapped by } \\
\text { other Ontolgoy }\end{array}$ & $\begin{array}{l}\text { field=value } \\
\text { in text file }\end{array}$ & Yes & Both \\
\hline ConceptNet & concept & Yes & $\begin{array}{l}\text { Inconsistency } \\
\text { allowed }\end{array}$ & Yes & Yes & WordNet & $\begin{array}{l}\text { Python, } \\
\text { XML }\end{array}$ & Yes & Semantic \\
\hline PurposeNet & purpose & No & Yes & Yes & Yes & WordNet & OWL & Yes & Semantic \\
\hline BabelNet & synset & No & Yes & No & Yes & WordNet & JAVA & Yes & Both \\
\hline PropBank & verb, arguments & No & Yes & No & Yes & $\begin{array}{l}\text { mapped by } \\
\text { VerbNet }\end{array}$ & text file & No & Semantic \\
\hline LifeNet & concept & No & Yes & Yes & Yes & $\begin{array}{c}\text { WordNet, } \\
\text { ConceptNet }\end{array}$ & $\begin{array}{l}\text { Python, } \\
\text { XML }\end{array}$ & No & Semantic \\
\hline MindNet & $\begin{array}{l}\text { dependency } \\
\text { graph }\end{array}$ & NO & Yes & Yes & No & WordNet & JAVA & Limited & Semantic \\
\hline
\end{tabular}




\section{CONCLUSION}

Various ontologies have been developed in the recent past. Some are domain dependent, discussing knowledge about a particular domain and some are upper ontology which store knowledge irrespective of any domain and is relevant for all the fields. We have discussed upper ontologies in this paper with varying underlying principle. Some of the ontologies contains lexicalized knowledge like WordNet, some are thoroughly semantic like ConceptNet. Some are available to all like PropBank and some are copyrighted and only limited version is available e.g Cyc. Ontologies like VerbNet are populated manually like PurposeNet and BabelNet is constructed from sources like Wikipedia. This is our humble attempt to collate different ontologies which contains domain-independent knowledge that are helpful in Natural Language Processing presenting different aspects of human language.

\section{REFERENCES}

[1]. Pease, I. Niles, J. Li, "The Suggested Upper Merged Ontology: A Large Ontology for the Semantic Web and its Applications", In Working Notes of the AAAI2002 Workshop on Ontologies and the Semantic Web.

[2]. Fellbaum (1998, ed.) WordNet: An Electronic Lexical Database. Cambridge, MA: MIT Press.

[3]. Collin F Baker, Charles J. Fillmore, John B. Lowe,"The Berkeley FrameNet Project" In Proc. COLING ' 98 Proceedings of the $17^{\text {th }}$ Intl. Conf. on Computational Linguistics- Vol1, pp 86-90.

[4]. Cycorp(2), "The CYC Technology", Cycorp, Inc, http://www.cyc.com

[5]. Fellbaum, Christiane, "WordNet and wordnets". In: Brown, Keith et al. (eds.), Encyclopedia of Language and Linguistics, Second Edition, Oxford: Elsevier, pp. 665-670, 2005.

[6]. George A. Miller,"WordNet: A Lexical Database for English", Communications of the ACM Vol. 38, No. 11, pp. 39-41, 1995.

[7]. Havasi, C.; Speer, R.; and Alonso, J.“ConceptNet 3: a flexible, multilingual semantic network for common sense knowledge". In Recent Advances in Natural LanguageProcessing, 2007.

[8]. Karin Kipper Schuler, "VerbNet: A broad-coverage, comprehensive verb lexicon" (January 1, 2005). Dissertations available from ProQuest. Paper AAI3179808. http://repository.upenn.edu/dissertations/AAI3179808

[9]. Lucy Vanderwende, Gary Kacmarcik, Hisami Suzuki, and Arul Menezes, MindNet: an automatically-created lexical resource, in HLT/EMNLP Interactive Demonstrations Proceedings, October 2005.

[10]. Martha Palmer, Dan Gildea, Paul Kingsbury, The Proposition Bank: A Corpus Annotated with Semantic Roles Computational Linguistics Journal, 31:1, 2005.

[11]. Paul Kingsbury and Martha Palmer. From Treebank to PropBank. 2002. In Proceedings of the 3rd International Conference on Language Resources and Evaluation (LREC-2002), Las Palmas, Spain.
[12]. Push Singh and William Williams (2003). LifeNet: a propositional model of ordinary human activity. Proceedings of the Workshop on Distributed and Collaborative Knowledge Capture (DC-KCAP) at KCAP 2003. Sanibel Island, FL.

[13]. R. Navigli and S. Ponzetto. BabelNet: The Automatic Construction, Evaluation and Application of a WideCoverage Multilingual Semantic Network. Artificial Intelligence, 193, Elsevier, 2012, pp. 217-250.

[14]. R. Sanghal, S. Paul, P.K. Mayee: PurposeNet: A knowledge Base Organized Around Purpose in Proc. 20th International Conference on Conceptual Structures, ICCS 2013, Mumbai, India, January 10-12, 2013, pp. 29-30.

[15]. Wei-Te Chen, Su-Chu Lin, Shu-Ling Huang, YouShan Chung, and Keh-Jiann Chen : E-HowNet and Automatic Construction of a Lexical Ontology in Proc. COLING ' 10 of $23^{\text {rd }}$ Intl. Conf. on Computational Linguistics: Demonstrations pp. 45-48.

[16].Z. Dong, Q. Dong, C. Hao: HowNet and its Computation of Meaning in Proc. Of COLING' 10 of $23^{\text {rd }}$ Intl. Conf. on Computational Linguistics: Demonstrations pp. 53-56. 